\title{
Kinematics and Dust Scattering in IRAS 09371+1212
}

\author{
J.P. Phillips ${ }^{1}$ and L. Cuesta ${ }^{2}$ \\ ${ }^{1}$ Instituto Nacional de Astrofísica, Óptica y Electrónica; ${ }^{2}$ Instituto de Astrofísica de Canarias
}

We have observed IRAS 09371+1212 using both narrow and broad band filters. As a result, we note that relative color trends B-V/V-R appear to be inconsistent with both the mean IS reddening law, and the variation deduced for model grains (Robinson et al, 1992). There also appears to be a disparity in the B-V/V-R reddening gradient as between the inner and outer source envelopes-a characteristic which, together with other evidence, may indicate a corresponding evolution in grain properties.

The interior lobes display particularly low values of $\mathbf{V} / \mathbf{R}$, with some evidence for asymmetric extensions in reddening towards the east. We propose that enhanced reddening of the northern lobe may arise from asymmetries in dust absorption within the lobes, or the combined effects of forwards grain scattering and source tilting to the line-of-sight.

Using the narrow $\mathrm{H} \alpha$ absorption feature in our spectroscopic observations, we find that line width and velocities in the northern lobe increase with increasing radial displacement in, whilst velocities in the southern lobe are more-or-less invariant.

We believe that such trends are indicative of the presence of both multiple scattering, and grain extinction and forward scattering. In particular, the last cited grain properties are shown to result in a reduction in the apparent Doppler-shifted grain scatter velocity. We obtain reasonable simulations of the observed trends using a combination of both effects. A further consequence is that grain velocities are found to be similar to gas outflow velocities deduced from $\mathrm{CO}$ spectroscopy.

\section{REFERENCES}

Dougados, C., Rouan, D., and Lena, P., 1992, Astron. Astrophys., 253, 464.

Robinson, G., Smith, R. G., and Hyland, A. R., 1992, Mon. Not. Roy. Astron. Soc., 256, 437.

Roddier, F., Roddier, C., Graves, J. E., and Northcott, M. J., 1995, Astrophys. J., 443, 249. 